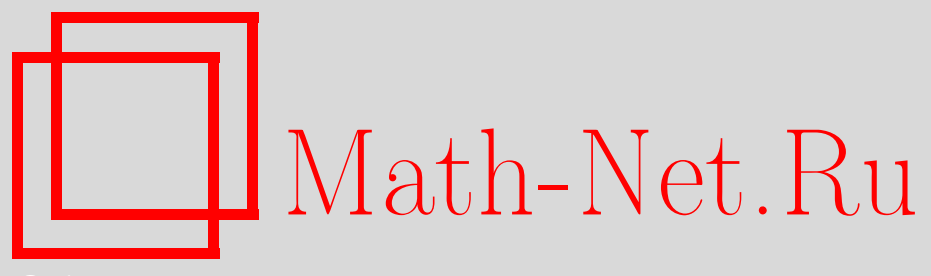

И. И. Скрыпник, Об устранимости изолированной особенности для анизотропных эллиптических уравнений с абсорбцией, Матем. сб., 2008, том 199, номер 7, 85102

DOI: https://doi.org/10.4213/sm3909

Использование Общероссийского математического портала Math-Net.Ru подразумевает, что вы прочитали и согласны с пользовательским соглашением http://www . mathnet.ru/rus/agreement

Параметры загрузки:

IP : 34.239 .49 .27

26 апреля 2023 г., $16: 52: 16$

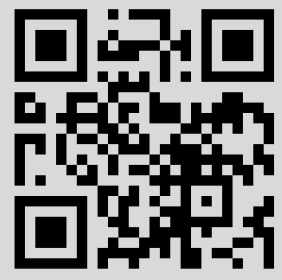




\section{И. И. Скрыпник}

\section{Об устранимости изолированной особенности для анизотропных эллиптических уравнений с абсорбцией}

Работа посвящена изучению решений с точечной особенностью для общего эллиптического уравнения вида

$$
-\sum_{i=1}^{n} \frac{\partial}{\partial x_{i}}\left(\left|\frac{\partial u}{\partial x_{i}}\right|^{p_{i}-2} \frac{\partial u}{\partial x_{i}}\right)+|u|^{q-1} u=0 .
$$

Развит метод получения новых поточечных оценок решения и интегральных оценок градиента решения. Установлены точные условия поведения члена уравнения, характеризующего абсорбцию, при которых не существует решений с точечной особенностью.

Библиография: 11 названий.

\section{$\S$ 1. Введение}

В настоящей работе изучается устранимость изолированных особенностей решений квазилинейного эллиптического уравнения

$$
-\sum_{i=1}^{n} \frac{d}{d x_{i}} a_{i}\left(x, u, \frac{\partial u}{\partial x}\right)+a_{0}\left(x, u, \frac{\partial u}{\partial x}\right)+g(x, u)=0
$$

в $\Omega \backslash\left\{x_{0}\right\}$, где $\Omega$ - ограниченная область в $\mathbb{R}^{n}, x_{0} \in \Omega$.

Предполагаем, что коэффициенты $g(x, u), a_{i}(x, u, \xi), i=0, \ldots, n$, определены при $x \in \Omega, u \in \mathbb{R}^{1}, \xi \in \mathbb{R}^{n}$, удовлетворяют условиям Каратеодори и при некоторых положительных постоянных $\nu_{1}, \nu_{2}$ справедливы неравенства

$$
\begin{gathered}
\sum_{i=1}^{n} a_{i}(x, u, \xi) \xi_{i} \geqslant \nu_{1} \sum_{i=1}^{n}\left|\xi_{i}\right|^{p_{i}}, \\
\left|a_{i}(x, u, \xi)\right| \leqslant \nu_{2}\left(\left(\sum_{j=1}^{n}\left|\xi_{j}\right|^{p_{j}}\right)^{1-\frac{1}{p_{i}}}+|u|^{p\left(1-\frac{1}{p_{i}}\right)}+1\right), \quad i=1, \ldots, n, \\
\left|a_{0}(x, u, \xi)\right| \leqslant \nu_{2}\left(\left(\sum_{j=1}^{n}\left|\xi_{j}\right|^{p_{j}}\right)^{1-\frac{1}{p}}+|u|^{p-1}+1\right), \\
g(x, u) \operatorname{sign} u \geqslant \nu_{1}\left(|u|^{q}-1\right), \quad|g(x, u)| \leqslant \nu_{2}\left(|u|^{q}+1\right),
\end{gathered}
$$

Работа выполнена при частичной финансовой поддержке фонда INTAS (грант № 051000008-7921).

(С) И. И. Скрыпник, 2008 
а числа $p, q, p_{i}, i=1, \ldots, n$, удовлетворяют условиям

$$
\begin{aligned}
1<p_{1} \leqslant p_{2} \leqslant \cdots \leqslant p_{n} & <\frac{n-1}{n-p} p, \quad \frac{1}{p}=\frac{1}{n} \sum_{i=1}^{n} \frac{1}{p_{i}}, \quad p<n, \\
q & \geqslant \frac{n(p-1)}{n-p} .
\end{aligned}
$$

Первый результат по устранимости изолированной особенности для решения уравнения

$$
-\Delta u+u^{q}=0, \quad x \in \Omega \backslash\left\{x_{0}\right\},
$$

был получен Х. Брезисом и Л. Вероном (см. [1]), которые показали, что в случае $q \geqslant n /(n-2)$ любое решение уравнения (1.5) имеет устранимую особенность в точке $\left\{x_{0}\right\}$.

Для квазилинейных эллиптических и параболических уравнений специального вида с абсорбцией аналогичные вопросы рассматривались многими авторами. Обзор полученных результатов и литературные ссылки можно найти в монографии Л. Верона [2].

Устранимость изолированных особенностей для общих эллиптических и параболических уравнений с абсорбцией получена автором в работах [3], [4].

Уравнения вида

$$
\sum_{i=1}^{n} \frac{\partial}{\partial x_{i}}\left(\left|\frac{\partial u}{\partial x_{i}}\right|^{p_{i}-2} \frac{\partial u}{\partial x_{i}}\right)+g(x)|u|^{p-2} u=0
$$

мало изучены.

Локальная ограниченность решений уравнения (1.6) была получена в работах [5], [6] при условии

$$
1<p_{1} \leqslant p_{2} \leqslant \cdots \leqslant p_{n} \leqslant \frac{n p}{n-p}, \quad p<n .
$$

М. Джаквинта (см. [7]) и П. Марчеллини (см. [8]) построили контрпримеры нерегулярных и неогранченных решений уравнения (1.6) при $p_{n}>n p /(n-p)$. В связи с тем, что не удается построить в явном виде фундаментальное решение уравнения (1.6), до недавнего времени не было понятно, как сформулировать точное условие устранимости изолированной особенности для решения этого уравнения. Этот вопрос был успешно решен в работе [9], где доказывается, что особенность в точке $\left\{x_{0}\right\}$ устранима, если $g(x) \in L_{\frac{n}{p-\delta}}(\Omega), 0<\delta<1$, и

$$
\underset{D(R) \backslash D(r)}{\operatorname{ess} \sup _{D}}|u(x)|=o\left(r^{-\frac{n-p}{p-1}}\right), \quad p<n,
$$

где $R$ - некоторое фиксированное число и

$$
\begin{aligned}
& D(r)=\left\{x \in \Omega:\left(\sum_{i=1}^{n}\left|x_{i}-x_{i}^{(0)}\right|^{\frac{a_{i}}{a_{1}}}\right)^{a_{1}} \leqslant r\right\}, \\
& a_{i}=\frac{p_{i}(p-1)}{p\left(p_{i}-1\right)+n\left(p-p_{i}\right)}, \quad i=1, \ldots, n .
\end{aligned}
$$


Условие (1.8) является точным и совпадает с аналогичным условием для уравнения (1.6) при $p_{1}=p_{2}=\cdots=p_{n}=p$ (см. [10]).

В настоящей работе доказывается, что если выполнены условия (1.3), (1.4), то изолированная особенность решения уравнения (1.1) устранима. Доказательство основано на новых априорных оценках решений с особенностью.

Метод получения подобных оценок был разработан в работе [11] и в дальнейшем развит в работах [3], [4], [9], [10].

В $\S 2$ мы формулируем вспомогательные утверждения и основные результаты. Вспомогательные поточечные интегральные оценки решения уравнения (1.1) доказываются в $\S 3,4$. Основная поточечная оценка решения уравнения (1.1) доказывается в 5 .

\section{§ 2. Вспомогательные утверждения и основные результаты}

Анизотропное пространство Соболева $W_{p_{1}, \ldots, p_{n}}^{1}(\Omega)$ будем обозначать через $W_{(p)}^{1}(\Omega)$.

Следующую теорему вложения можно найти, например, в [5].

ЛЕмма 2.1. Пусть $u(x) \in \stackrel{\circ}{W}_{1}^{1}(\Omega) u$

$$
\sum_{i=1}^{n} \int_{\Omega}|u(x)|^{\alpha_{i}}\left|\frac{\partial u}{\partial x_{i}}\right|^{p_{i}} d x<\infty, \quad \alpha_{i} \geqslant 0, p_{i} \geqslant 1 .
$$

Если $1<p<n$, mo

$$
u(x) \in L_{q}(\Omega), \quad q=\frac{n p}{n-p}\left(1+\frac{1}{n} \sum_{i=1}^{n} \frac{\alpha_{i}}{p_{i}}\right)
$$

и выполнено следующее неравенство:

$$
\|u\|_{L_{q}(\Omega)} \leqslant c \prod_{i=1}^{n}\left(\int_{\Omega}|u(x)|^{\alpha_{i}}\left|\frac{\partial u}{\partial x_{i}}\right|^{p_{i}} d x\right)^{\frac{1}{n p_{i}\left(1+\frac{1}{n} \sum_{k=1}^{n} \frac{\alpha_{k}}{p_{k}}\right)}},
$$

где с - некоторая положительная постоянная, зависящая лишь от $n, \alpha_{i}, p_{i}$, $i=1, \ldots, n$.

В дальнейшем также будем использовать следующее простое утверждение, доказательство которого можно найти в $[11 ;$ гл. $8, \S 1]$.

Лемма 2.2. Пусть $\left\{\alpha_{i}\right\}, i=1,2, \ldots,-$ ограниченная числовая последовательность такая, что для всех $j \geqslant 1$ выполнено неравенство

$$
\alpha_{j} \leqslant A a^{j} \alpha_{j+1}^{\sigma}, \quad \sigma \in(0,1), \quad A, a \geqslant 1 .
$$

Тогда

$$
\alpha_{1} \leqslant c A^{\frac{1}{1-\sigma}}
$$

где постоянная с зависит лишь от $\sigma, a$. 
Определим понятие сингулярного решения уравнения (1.1) с особенностью в точке $\left\{x_{0}\right\}$. Под решением уравнения (1.1) с особенностью в $\left\{x_{0}\right\}$ понимаем функцию $u(x)$, удовлетворяющую включению

$$
u(x) \psi(x) \in W_{(p)}^{1}(\Omega)
$$

и интегральному тождеству

$$
\sum_{i=1}^{n} \int_{\Omega} a_{i}\left(x, u, \frac{\partial u}{\partial x}\right) \frac{\partial \varphi}{\partial x_{i}} d x+\int_{\Omega} a_{0}\left(x, u, \frac{\partial u}{\partial x}\right) \varphi d x+\int_{\Omega} g(x, u) \varphi d x=0
$$

с $\varphi(x)=\psi(x) \zeta(x)$, где $\psi(x)$ - произвольная функция класса $C^{\infty}(\Omega)$, равная нулю в окрестности $\left\{x_{0}\right\}$, и $\zeta(x)$ - произвольная функция из пространства $\stackrel{\circ}{W}_{(p)}^{1}(\Omega)$.

Будем говорить, что сингулярность в точке $\left\{x_{0}\right\}$ устранима, если $u(x) \in$ $W_{(p)}^{1}(\Omega)$ и интегральное тождество (2.5) справедливо для любой функции $\varphi(x) \in \stackrel{\circ}{W}_{(p)}^{1}(\Omega)$.

Нашим основным результатом является следующая

Теорема 2.1. Пусть $и(x)$ является сингулярным решением уравнения (1.1) в области $\Omega \backslash\left\{x_{0}\right\}$ и выполнены условия (1.2)-(1.4). Тогда особенность в точке $\left\{x_{0}\right\}$ устранима.

Доказательство теоремы 2.1 состоит из нескольких этапов. Вначале устанавливается предварительная поточечная оценка, которая дается следующей теоремой.

ТеОрема 2.2. Пусть выполнены все условия теоремы 2.1. Тогда существует постоянная $K_{1}$, зависящая лишь от $\nu_{1}, \nu_{2}, q, n, p_{i}, i=1, \ldots, n$, такая, что справедлива ощенка

$$
|u(x)| \leqslant K_{1}\left(\sum_{i=1}^{n}\left|x_{i}-x_{i}^{(0)}\right|^{\frac{p_{i} q}{q-p_{i}+1}}\right)^{-\frac{1}{q}}
$$

$\partial л я$

$$
0<\sum_{i=1}^{n}\left|x_{i}-x_{i}^{(0)}\right|^{\frac{p_{i} q}{q-p_{i}+1}} \leqslant R_{0}, \quad x \in \Omega .
$$

В дальнейшем, используя (2.6) и некоторые интегральные оценки градиента из $\S 4$, мы получаем улучшенную оценку вида

$$
|u(x)| \leqslant K_{2}\left(\sum_{i=1}^{n}\left|x_{i}-x_{i}^{(0)}\right|^{a_{i}}\right)^{-\frac{n-p}{p-1}+\theta}, \quad \theta>0,
$$

для $0<\sum_{i=1}^{n}\left|x_{i}-x_{i}^{(0)}\right|^{a_{i}} \leqslant R_{0}, a_{i}$ определены в (1.10). Из (2.7) мы получим, что решение $u(x)$ удовлетворяет включению

$$
|u(x)|^{q-p+1} \in L_{\frac{n}{p-\delta_{0}}}(\Omega)
$$

с некоторым $0<\delta_{0}<1$, поэтому теорема 2.1 является следствием результатов работы [9]. 
ЗАмЕчАниЕ. Все результаты работы остаются справедливыми для более общего уравнения, когда справедливы структурные неравенства

$$
\begin{gathered}
\sum_{i=1}^{n} a_{i}(x, u, \xi) \xi_{i} \geqslant \nu_{1} \sum_{i=1}^{n}\left|\xi_{i}\right|^{p_{i}} \\
\left|a_{i}(x, u, \xi)\right| \leqslant \nu_{2}\left(\sum_{j=1}^{n}\left|\xi_{j}\right|^{p_{j}}\right)^{1-\frac{1}{p_{i}}}+g_{1}(x)|u|^{p\left(1-\frac{1}{p_{i}}\right)}+f_{1}(x), \quad i=1, \ldots, n, \\
\left|a_{0}(x, u, \xi)\right| \leqslant h(x)\left(\sum_{j=1}^{n}\left|\xi_{j}\right|^{p_{j}}\right)^{1-\frac{1}{p}}+g_{2}(x)|u|^{p-1}+f_{2}(x), \\
g(x, u) \operatorname{sign} u \geqslant \nu_{1}|u|^{q}-f_{3}(x), \quad|g(x, u)| \leqslant \nu_{2}|u|^{q}+f_{4}(x)
\end{gathered}
$$

с неотрицательными функциями $h(x), g_{1}(x), g_{2}(x), f_{1}(x)-f_{4}(x)$, для которых справедливо включение

$$
\begin{gathered}
F(x) \in L_{\frac{n}{p-\delta}}(\Omega), \quad 0<\delta<1, \\
F(x)=h^{p}(x)+\sum_{i=1}^{n}\left[f_{1}(x)\right]^{\frac{p_{i}}{p_{i}-1}}+\sum_{i=1}^{n}\left[g_{1}(x)\right]^{\frac{p_{i}}{p_{i}-1}}+g_{2}(x)+f_{2}(x)+f_{3}(x)+f_{4}(x) .
\end{gathered}
$$

\section{§ 3. Доказательство теоремы 2.2}

Без ограничения общности считаем, что $x_{0}=0$. Зафиксируем положительное число

$$
R_{0} \leqslant \frac{1}{2} \min \{1, \operatorname{dist}(0, \partial \Omega)\}
$$

и обозначим для $0<r \leqslant R_{0}$

$$
\begin{gathered}
\Omega(r)=\left\{x \in \Omega:\left(\sum_{i=1}^{n}\left|x_{i}\right|^{\bar{p}_{i}}\right)^{\frac{1}{a}} \leqslant r\right\}, \\
a=\frac{p q}{q-p+1}, \quad \bar{p}_{i}=\frac{p_{i} q}{q-p_{i}+1}, \quad i=1, \ldots, n, \\
M(r)=\operatorname{ess} \sup \left\{|u(x)|: x \in \Omega\left(R_{0}\right) \backslash \Omega(r)\right\} .
\end{gathered}
$$

Используя итерационный метод Мозера, легко показать, что величина $M(r)$ конечна при любом $r>0$. Далее предполагаем, что $\lim _{r \rightarrow 0} M(r)=\infty$, так как в противном случае устранимость особенности следует из работы [9]. Для $0<\rho<R_{0} / 4, \sigma \in(0,1 / 8)$ рассмотрим функцию

$$
\varphi_{\sigma \rho}(x)=\omega\left(\frac{\left(\sum_{i=1}^{n}\left|x_{i}\right|^{\bar{p}_{i}}\right)^{\frac{1}{a}}}{\rho}\right),
$$

где $\omega: \mathbb{R}^{1} \rightarrow \mathbb{R}^{1}-$ функция класса $C^{\infty}$ такая, что $\omega(s)=1$ при $1 \leqslant s \leqslant 2$, $\omega(s)=0$ вне интервала $1-\sigma \leqslant s \leqslant 2+\sigma ; 0 \leqslant \omega(s) \leqslant 1,\left|\frac{d \omega(s)}{d s}\right| \leqslant \frac{2}{\sigma}$.

Будем обозначать через $\gamma$ всевозможные постоянные, зависящие лишь от $\nu_{1}$, $\nu_{2}, n, p_{1}, \ldots, p_{n}$. 
ЛЕмма 3.1. Предположим, что выполнены все условия теоремы 2.1. Тогда справедливо неравенство

$$
\sum_{i=1}^{n} \int_{\Omega}\left|\frac{\partial u}{\partial x_{i}}\right|^{p_{i}} \varphi_{\sigma \rho}^{k}(x) d x+\int_{\Omega}|u|^{q+1} \varphi_{\sigma \rho}^{k}(x) d x \leqslant \gamma \sigma^{-\frac{p(p+1)}{q-p+1}} \rho^{n-\frac{p(q+1)}{q-p+1}}
$$

здесъ $k$ - фиксированное положительное число, $k \geqslant p_{n}$.

ДокАЗАтЕЛьство. Подставим в интегральное тождество (2.5) функции

$$
\psi(x)=u(x) \varphi_{\sigma \rho}^{k-1}(x), \quad \zeta(x)=\varphi_{\sigma \rho}(x) .
$$

Используя условия (1.2), получим

$$
\begin{aligned}
& \sum_{i=1}^{n} \int_{\Omega}\left|\frac{\partial u}{\partial x_{i}}\right|^{p_{i}} \varphi_{\sigma \rho}^{k}(x) d x+\int_{\Omega}|u(x)|^{q+1} \varphi_{\sigma \rho}^{k}(x) d x \\
& \quad \leqslant \gamma \sum_{i=1}^{n}(\sigma \rho)^{-\frac{p\left(q-p_{i}+1\right)}{q-p+1}} \int_{\Omega}|u(x)|^{p_{i}} \varphi_{\sigma \rho}^{k-p_{n}}(x) d x+\gamma \int_{\Omega}\left(1+|u(x)|^{p}\right) \varphi_{\sigma \rho}^{k-p_{n}}(x) d x .
\end{aligned}
$$

Отметим, что в силу условий (1.3), (1.4) выполнено неравенство

$$
p_{1} \leqslant p \leqslant p_{n}<q+1
$$

поэтому неравенство Юнга влечет

$$
\begin{aligned}
& \gamma \sum_{i=1}^{n}(\sigma \rho)^{-\frac{p\left(q-p_{i}+1\right)}{q-p+1}} \int_{\Omega}|u(x)|^{p_{i}} \varphi_{\sigma \rho}^{k-p_{n}}(x) d x \\
& \quad \leqslant \frac{1}{4} \int_{\Omega}|u(x)|^{q+1} \varphi_{\sigma \rho}^{k}(x) d x+\gamma \sigma^{-\frac{p(q+1)}{q-p+1}} \rho^{n-\frac{p(q+1)}{q-p+1}}
\end{aligned}
$$

Здесь мы также воспользовались очевидным неравенством

$$
\operatorname{mes} \Omega(\rho) \leqslant \gamma \rho^{n} \text {. }
$$

Аналогичным образом оценивается второе слагаемое правой части (3.4). Оценка (3.3) является следствием неравенств (3.4), (3.6). Таким образом, лемма 3.1 доказана.

Подставим в интегральное тождество (2.5) пробные функции

$$
\psi(x)=u(x)|u(x)|^{l} \varphi_{\sigma \rho}^{k-1}(x), \quad \zeta(x)=\varphi_{\sigma \rho}(x), \quad k, l>0 .
$$

Используя условия (1.2), получим

$$
\begin{gathered}
\sum_{i=1}^{n} \int_{\Omega}|u(x)|^{l}\left|\frac{\partial u}{\partial x_{i}}\right|^{p_{i}} \varphi_{\sigma \rho}^{k}(x) d x+\int_{\Omega}|u(x)|^{l+q+1} \varphi_{\sigma \rho}^{k}(x) d x \\
\leqslant \gamma(k+l)^{p_{n}} \sum_{i=1}^{n}(\sigma \rho)^{-p \frac{q-p_{i}+1}{q-p+1}} \int_{\Omega}|u(x)|^{l+p_{i}} \varphi_{\sigma \rho}^{k-p_{n}}(x) d x \\
+\gamma(k+l)^{p_{n}} \int_{\Omega}\left(1+|u(x)|^{p}\right)|u(x)|^{l} \varphi_{\sigma \rho}^{k-p_{n}}(x) d x .
\end{gathered}
$$


Положим

$$
H(\rho)=1+M^{p}(\rho)+\sum_{i=1}^{n} \rho^{-p \frac{q-p_{i}+1}{q-p+1}} M^{p_{i}}(\rho) ;
$$

тогда неравенство (3.8) влечет

$$
\begin{aligned}
& \sum_{i=1}^{n} \int_{\Omega}|u(x)|^{l}\left|\frac{\partial u}{\partial x_{i}}\right|^{p_{i}} \varphi_{\sigma \rho}^{k}(x) d x+\int_{\Omega}|u(x)|^{l+q+1} \varphi_{\sigma \rho}^{k}(x) d x \\
& \quad \leqslant \gamma(k+l)^{p_{n}} \sigma^{-p \frac{q-p_{1}+1}{q-p+1}} H(\rho(1-\sigma)) M^{p_{n}}(\rho(1-\sigma)) \int_{\Omega}|u(x)|^{l-p_{n}} \varphi_{\sigma \rho}^{k-p_{n}}(x) d x .
\end{aligned}
$$

Пусть $l, l_{1}, l_{2}$ - произвольные положительные числа такие, что

$$
l=l_{1}+l_{2}, \quad l_{1}-p=l_{2} \frac{n}{p}-q-1 .
$$

Используя неравенство Гёльдера, неравенство (3.9) и лемму 2.1 при $\alpha_{1}=\cdots=$ $\alpha_{n}=l_{1}-p$, получим

$$
\begin{aligned}
\int_{\Omega}|u(x)|^{l} \varphi_{\sigma \rho}^{l}(x) d x & \\
\leqslant & \left(\int_{\Omega}\left[|u(x)| \varphi_{\sigma \rho}(x)\right]^{l_{1} \frac{n}{n-p}} d x\right)^{\frac{n-p}{n}}\left(\int_{\Omega}\left[|u(x)| \varphi_{\sigma \rho}(x)\right]^{l_{2} \frac{n}{p}} d x\right)^{\frac{p}{n}} \\
\leqslant & \prod_{i=1}^{n}\left(\int_{\Omega}\left(|u(x)| \varphi_{\sigma \rho}(x)\right)^{l_{1}-p}\left|\frac{\partial}{\partial x_{i}}\left(u(x) \varphi_{\sigma \rho}(x)\right)\right|^{p_{i}} d x\right)^{\frac{p}{n p_{i}}} \\
& \times\left(\int_{\Omega}\left[|u(x)| \varphi_{\sigma \rho}(x)\right]^{l_{2} \frac{n}{p}} d x\right)^{\frac{p}{n}} \\
\leqslant & \gamma l^{p_{n}\left(1+\frac{p}{n}\right)} \sigma^{-p \frac{q-p_{1}+1}{q-p_{1}}\left(1+\frac{p}{n}\right)} H^{1+\frac{p}{n}}(\rho(1-\sigma)) M^{\left(1+\frac{p}{n}\right) p_{n}}(\rho(1-\sigma)) \\
& \times\left(\int_{\Omega}\left[|u(x)| \varphi_{\sigma \rho}(x)\right]^{l \frac{n}{n+p}}-(q+1-p) \frac{p}{n+p}-p-p_{n}\right)^{1+\frac{p}{n}} \cdot
\end{aligned}
$$

Рассмотрим последовательность $\left\{l_{j}\right\}$, определенную следующим образом:

$l_{j}=\left(2(q+1)+n+p_{n} \frac{n+p}{p}\right)\left(\frac{n+p}{n}\right)^{j}-q-1-n-p_{n} \frac{n+p}{p}, \quad j=0,1,2, \ldots$.

Используя итерационный метод Мозера, из (3.11) получим

$$
\begin{aligned}
& M^{2(q+1)+n+p_{n} \frac{n+p}{p}}(\rho) \\
& \quad \leqslant \gamma \sigma^{-\gamma} H^{1+\frac{n}{p}}(\rho(1-\sigma)) M^{p_{n}\left(1+\frac{n}{p}\right)}(\rho(1-\sigma)) \int_{\Omega}\left[|u(x)| \varphi_{\sigma \rho}(x)\right]^{q+1} d x .
\end{aligned}
$$

Положим

$$
y(\rho)=M(\rho) \rho^{\frac{p}{q-p+1}} .
$$

Тогда, используя лемму 3.1, из (3.12) получим

$$
y^{2(q+1)+n+p_{n}\left(1+\frac{n}{p}\right)}(\rho) \leqslant \gamma \sigma^{-\gamma} y^{p_{n}\left(1+\frac{n}{p}\right)}(\rho(1-\sigma)) H_{1}^{1+\frac{n}{p}}(\rho(1-\sigma)),
$$


где

$$
H_{1}(\rho)=\rho^{p \frac{q+1}{q-p+1}}+\rho^{p} y^{p}(\rho)+\sum_{i=1}^{n} y^{p_{i}}(\rho) .
$$

Выберем последовательность $\left\{\sigma_{j}\right\}, j=1,2, \ldots, \sigma_{j}=2^{-(j-1)}$, и обозначим

$$
y_{j}=y\left(\rho-\left(\sigma_{1}+\cdots+\sigma_{j}\right) \rho\right), \quad H_{j}=\rho^{p \frac{q+1}{q-p+1}}+\rho^{p} y_{j}^{p}+\sum_{i=1}^{n} y_{j}^{p_{i}} .
$$

Из неравенства (3.13) следует оценка

$$
\begin{gathered}
y_{j} \leqslant \gamma 2^{j \gamma} H_{j+1}^{r_{0}} y_{j+1}^{r_{1}}, \quad j=1,2, \ldots, \\
r_{0}=\frac{n+p}{p} \frac{1}{2(q+1)+n+p_{n} \frac{n+p}{p}}, \quad r_{1}=p_{n} r_{0} .
\end{gathered}
$$

Условия (1.3), (1.4) обеспечивают неравенство $2 r_{1}<1$. Используя лемму 2.2 , из (3.14) получим

$$
y_{1}=M(\rho) \rho^{\frac{p}{q-p+1}} \leqslant \gamma,
$$

что и доказывает теорему 2.2 .

\section{§ 4. Интегральные оценки решения}

Определим при $0 \leqslant \lambda<n-p$ числа

$$
\begin{gathered}
b_{i}(\lambda)=\frac{p_{i}}{p+\frac{n-p-\lambda}{p-1}\left(p-p_{i}\right)}, \quad i=1,2, \ldots, n, \\
b_{i}(\lambda)>0, \quad b_{i}(0)=a_{i}, \quad i=1,2, \ldots, n,
\end{gathered}
$$

где $a_{i}$ определены в (1.10).

Рассмотрим при $r \in\left(0, R_{0}\right)$ функцию

$$
\begin{gathered}
\psi_{r}: \mathbb{R}^{n} \rightarrow \mathbb{R}^{1}, \quad \psi_{r}(x)=\eta_{r}\left(\left(\sum_{i=1}^{n}\left|x_{i}\right|^{\frac{b_{i}(\lambda)}{b_{1}(\lambda)}}\right)^{b_{1}(\lambda)}\right), \\
\eta_{r}(s)= \begin{cases}0 & \text { при } s \leqslant r, \\
1 & \text { при } s \geqslant R(r), \\
-\left[(1-\theta) \ln \ln \frac{1}{r}\right]^{-1} \int_{r}^{s} \frac{1}{z \ln z} d z & \text { при } r<s<R(r),\end{cases}
\end{gathered}
$$

$\theta$ - выбираемое далее число из интервала $(0,1), R(r)$ определяется равенством

$$
\ln \frac{1}{R(r)}=\left[\ln \frac{1}{r}\right]^{\theta}
$$

Обозначим

$$
\begin{aligned}
& \widetilde{D}(r, \lambda)=\left\{x \in \Omega:\left(\sum_{i=1}^{n}\left|x_{i}\right|^{\frac{b_{i}(\lambda)}{b_{1}(\lambda)}}\right)^{b_{1}(\lambda)} \leqslant r\right\}, \\
& E(r, \lambda)=\{x \in \Omega \backslash\{0\}: u(x) \geqslant \widetilde{M}(r, \lambda)\},
\end{aligned}
$$




$$
\begin{aligned}
& \widetilde{M}(r, \lambda)=\operatorname{ess} \sup \left\{|u(x)|: x \in \widetilde{D}\left(R_{0}, \lambda\right) \backslash \widetilde{D}(r, \lambda)\right\}, \\
& u_{r}(x, \lambda)=(u(x)-\widetilde{M}(r, \lambda))_{+} .
\end{aligned}
$$

Определим функцию $F_{1}(r, \lambda)$ при $0<r<R_{0}, 0 \leqslant \lambda<n-p$ равенствами

$$
F_{1}(r, 0)= \begin{cases}{\left[\ln \frac{1}{r}\right]^{1-\frac{p_{1}-1}{q-p_{1}+1}}(r, \lambda)=R^{\lambda}(r) \quad \text { при } \lambda>0,} \\ \ln \ln \frac{1}{r} & \text { при } \frac{p_{1}-1}{q-p_{1}+1}<1, \\ {\left[\ln \frac{1}{R(r)}\right]^{1-\frac{p_{1}-1}{q-p_{1}+1}}} & \text { при } \frac{p_{1}-1}{q-p_{1}+1}=1, \quad q=\frac{n(p-1)}{n-p} . \\ & \text { при } \frac{p_{1}-1}{q-p_{1}+1}>1,\end{cases}
$$

Для упрощения записи в дальнейшем будем писать $b_{i}, E(r), \widetilde{M}(r), u_{r}(x)$ вместо $b_{i}(\lambda), E(r, \lambda), \widetilde{M}(r, \lambda), u_{r}(x, \lambda)$.

ЛЕмма 4.1. Предположим, что выполнены все условия теоремы 2.1, и пусть и $(x)$ дополнительно удовлетворяет неравенству

$$
|u(x)| \leqslant K^{\prime}\left(\sum_{i=1}^{n}\left|x_{i}\right|^{b_{i}}\right)^{-\frac{n-p-\lambda}{p-1}} \quad \text { npu } \quad 0<\sum_{i=1}^{n}\left|x_{i}\right|^{b_{i}}<R_{0} .
$$

Тогда при $r \in\left(0, R_{0}\right), R(r)<\rho<R_{0}, k>p_{n}$ справедлива оценка

$$
\begin{aligned}
& \sum_{i=1}^{n} \int_{E(\rho)} \frac{1}{u(x)}\left|\frac{\partial u}{\partial x_{i}}\right|^{p_{i}} \psi_{r}^{k}(x) d x+\int_{E(\rho)} \ln \frac{u(x)}{\widetilde{M}(\rho)} u^{q}(x) \psi_{r}^{k}(x) d x \\
& \leqslant K_{3}\left\{\left(\ln \ln \frac{1}{r}\right)^{-\bar{p}_{1}} F_{1}(r, \lambda)+\rho^{p+\lambda} \ln ^{p} \frac{1}{\rho}\right\}
\end{aligned}
$$

с постоянной $K_{3}$, зависящей лищъ от $K^{\prime}, \theta, \nu_{1}, \nu_{2}, n, p_{1}, \ldots, p_{n}, q$.

ДокАЗАтЕльство. Подставим в интегральное тождество (2.5) функции

$$
\psi(x)=\ln _{+} \frac{u(x)}{\widetilde{M}(\rho)} \psi_{r}^{k-1}(x), \quad \zeta(x)=\psi_{r}(x) .
$$

Используя неравенства (1.2), стандартными рассуждениями получим

$$
\begin{gathered}
\sum_{i=1}^{n} \int_{E(\rho)} \frac{1}{u(x)}\left|\frac{\partial u}{\partial x_{i}}\right|^{p_{i}} \psi_{r}^{k}(x) d x+\int_{E(\rho)} \ln \frac{u(x)}{\widetilde{M}(\rho)} u^{q}(x) \psi_{r}^{k}(x) d x \\
\leqslant \gamma \sum_{i=1}^{n} \int_{E(\rho)} u^{p_{i}-1}(x) \ln ^{p_{i}} \frac{u(x)}{\widetilde{M}(\rho)}\left|\frac{\partial \psi_{r}(x)}{\partial x_{i}}\right|^{p_{i}} \psi_{r}^{k-p_{n}}(x) d x \\
\quad+\gamma \int_{E(\rho)} u^{p-1}(x) \ln ^{p} \frac{u(x)}{\widetilde{M}(\rho)} \psi_{r}^{k-p_{n}}(x) d x=I_{1}+I_{2} .
\end{gathered}
$$


При $\lambda=0$, используя неравенство Юнга, имеем

$$
\begin{aligned}
I_{1}- & \frac{1}{4} \int_{E(\rho)} \ln \frac{u(x)}{\widetilde{M}(\rho)} u^{q}(x) \psi_{r}^{k}(x) d x \\
& \leqslant \gamma \sum_{i=1}^{n} \int_{E(\rho)}\left[\ln \frac{u(x)}{\widetilde{M}(\rho)}\right]^{\left(p_{i}-\frac{p_{i}-1}{q}\right) \frac{q}{q-p_{i}+1}}\left|\frac{\partial \psi_{r}(x)}{\partial x_{i}}\right|^{\bar{p}_{i}} d x .
\end{aligned}
$$

Положим

$$
x_{i}=\operatorname{sign} y_{i}\left|y_{i}\right|^{\varkappa \frac{b_{1}}{b_{i}}}, \quad \varkappa=2 \max _{1 \leqslant i \leqslant n} \frac{b_{i}}{b_{1}} .
$$

Тогда неравенство (4.5) при $\lambda=0$ влечет

$$
\begin{aligned}
& \sum_{i=1}^{n} \int_{E(\rho)}\left[\ln \frac{u(x)}{\widetilde{M}(\rho)}\right]^{\left(p_{i}-\frac{p_{i}-q}{q}\right) \frac{q}{q-p_{i}+1}}\left|\frac{\partial \psi_{r}(x)}{\partial x_{i}}\right|^{\bar{p}_{i}} d x \\
& \quad \leqslant \gamma \sum_{i=1}^{n}\left(\ln \ln \frac{1}{r}\right)^{-\bar{p}_{i}} \int_{\widetilde{D}(R(r)) \backslash \widetilde{D}(r)} \frac{\left|x_{i}\right|^{\left(\frac{b_{i}}{b_{1}}-1\right) \bar{p}_{i}}\left(\sum_{k=1}^{n}\left|x_{k}\right|^{\frac{b_{k}}{b_{1}}}\right)^{-\bar{p}_{i}}}{\left[\ln \frac{1}{\sum_{k=1}^{n}\left|x_{k}\right|^{b_{k} / b_{1}}}\right]^{\frac{p_{i}-1}{q-p_{i}+1}}} d x \\
& \quad \leqslant \gamma \sum_{i=1}^{n}\left(\ln \ln \frac{1}{r}\right)^{-\bar{p}_{i}} \int_{D_{1}(R(r)) \backslash D_{1}(r)} \frac{\left(\sum_{k=1}^{n}\left|y_{k}\right|^{\varkappa}\right)^{-\frac{b_{1}}{b_{i}} \bar{p}_{i}} \prod_{k=1}^{n}\left|y_{k}\right|^{\varkappa \frac{b_{1}}{b_{k}}-1}}{\left[\ln \frac{1}{|y|}\right]^{\frac{p_{i}-1}{q-p_{i}+1}}} d y \\
& \quad \leqslant \gamma\left(\ln \ln \frac{1}{r}\right)^{-\bar{p}_{1}} F_{1}(r, 0) .
\end{aligned}
$$

Здесь мы использовали обозначение

$$
D_{1}(r)=\left\{\left[\sum_{i=1}^{n}\left|y_{i}\right|^{\varkappa}\right]^{b_{1}} \leqslant r\right\} .
$$

При $\lambda>0$, используя неравенство (4.5), получим

$$
I_{1} \leqslant \sum_{i=1}^{n} \int_{\widetilde{D}(R(r)) \backslash \widetilde{D}(r)}\left(\sum_{k=1}^{n}\left|x_{k}\right|^{\frac{b_{k}}{b_{1}}}\right)^{-\frac{n-p-\lambda}{p-1}\left(p_{i}-1\right) b_{1}-p_{i}}\left|x_{i}\right|^{\left(\frac{b_{i}}{b_{1}}-1\right) p_{i}} d x \leqslant \gamma F_{1}(r, \lambda) .
$$

Аналогичным образом, используя неравенство (4.5) при $\lambda \geqslant 0$, получим

$$
I_{2} \leqslant \int_{\widetilde{D}(\rho)}\left(\sum_{i=1}^{n}\left|x_{i}\right|^{\frac{b_{i}}{b_{1}}}\right)^{(p-n+\lambda) b_{1}} \ln ^{p} \frac{1}{\sum_{i=1}^{n}\left|x_{i}\right|^{b_{i}}} d x \leqslant \gamma \rho^{p+\lambda} \ln ^{p} \frac{1}{\rho} .
$$

Здесь мы воспользовались также включением $E(\rho) \subset \widetilde{D}(\rho)$.

Собирая неравенства (4.7)-(4.11), получим (4.6). Таким образом, лемма 4.1 доказана.

Определим при $z \in \mathbb{R}^{1}$ функцию

$$
\Phi_{\rho}(z)=\left[(\widetilde{M}(\rho)-\widetilde{M}(2 \rho))^{1-\varepsilon}-(z-\widetilde{M}(2 \rho))^{1-\varepsilon}\right]_{+},
$$

где $\varepsilon-$ произвольное число из интервала $(1, n /(n-p))$. 
Обозначим при $0<r<R_{0}$

$$
F_{2}(r, \lambda)= \begin{cases}{[R(r)]^{\frac{\lambda}{p_{1}-1}}} & \text { при } \lambda>0, \\ {\left[\ln \frac{1}{R(r)}\right]^{-1}} & \text { при } \lambda=0 .\end{cases}
$$

ЛЕмма 4.2. Предположим, что выполнено все условия леммы 4.1. Тогда при $R(r)<\rho<R_{0}$ справедливо неравенство

$$
\sum_{i=1}^{n} \int_{E(\rho)}(u(x)-\widetilde{M}(2 \rho))^{-\varepsilon}\left|\frac{\partial u}{\partial x_{i}}\right|^{p_{i}} \psi_{r}^{k}(x) d x \leqslant K_{4} H(r, \rho)
$$

с постоянной $K_{4}$, зависящей лищъ от $K^{\prime}, \nu_{1}, \nu_{2}, n, p_{1}, \ldots, p_{n}$. Здесъ

$$
\begin{aligned}
H(r, \rho)=(\widetilde{M}(\rho)-\widetilde{M}(2 \rho))^{1-\varepsilon} & \times\left\{F_{2}^{p_{1}-1}(r, \lambda)+(\widetilde{M}(\rho)-\widetilde{M}(2 \rho))^{(1-\varepsilon)(p-1)} \rho^{n-\varepsilon(n-p)+\lambda}+\rho^{p+\lambda}\right. \\
& \left.+\sum_{i=1}^{n}\left\{\left[\ln \ln \frac{1}{r}\right]^{-\bar{p}_{1}} F_{1}(r)+\rho^{p+\lambda} \ln ^{p} \frac{1}{\rho}\right\}^{\frac{p_{i}-1}{p_{i}}} F_{2}^{\frac{p_{i}-1}{p_{i}}}(r, \lambda)\right\} .
\end{aligned}
$$

ДокАЗАТЕЛьСтво. Подставим в интегральное тождество (2.5) функции

$$
\psi(x)=\Phi_{\rho}(u(x)) \psi_{r}^{k-1}(x), \quad \zeta(x)=\psi_{r}(x) .
$$

Совершая стандартные преобразования, получим

$$
\begin{aligned}
& \sum_{i=1}^{n} \int_{E(\rho)}(u(x)-\widetilde{M}(2 \rho))^{-\varepsilon}\left|\frac{\partial u}{\partial x_{i}}\right|^{p_{i}} \psi_{r}^{k}(x) d x+\int_{E(\rho)} \Phi_{\rho}(u(x)) u^{q}(x) \psi_{r}^{k}(x) d x \\
& \quad \leqslant \gamma(\widetilde{M}(\rho)-\widetilde{M}(2 \rho))^{1-\varepsilon}\left\{I_{3}+I_{4}+I_{5}+I_{6}\right\}
\end{aligned}
$$

где

$$
\begin{gathered}
I_{3}=\sum_{i=1}^{n}\left(\int_{E(\rho)} \sum_{j=1}^{n}\left|\frac{\partial u}{\partial x_{j}}\right|^{p_{j}}\right)^{1-\frac{1}{p_{i}}}\left|\frac{\partial \psi_{r}(x)}{\partial x_{i}}\right| \psi_{r}^{k-1}(x) d x \\
I_{4}=\int_{E(\rho)}\left(\sum_{i=1}^{n}\left|\frac{\partial u}{\partial x_{i}}\right|^{p_{i}}\right)^{1-\frac{1}{p}} \psi_{r}^{k}(x) d x, \\
I_{5}=\sum_{i=1}^{n} \int_{E(\rho)} u^{p_{i}-1}(x)\left|\frac{\partial \psi_{r}(x)}{\partial x_{i}}\right|^{p_{i}} d x \\
I_{6}=\int_{E(\rho)} u^{p-1}(x) \psi_{r}^{k}(x) d x .
\end{gathered}
$$

Используя неравенство (4.5), получим

$$
\begin{aligned}
& \int_{E(\rho)} u^{p_{i}-1}(x)\left|\frac{\partial \psi_{r}(x)}{\partial x_{i}}\right|^{p_{i}} d x \\
& \leqslant \gamma\left[\ln \ln \frac{1}{r}\right]^{-p_{i}} \int_{\widetilde{D}(R(r)) \backslash \widetilde{D}(r)}\left(\sum_{k=1}^{n}\left|x_{k}\right|^{\frac{b_{k}}{b_{1}}}\right)^{-b_{1} \frac{n-p-\lambda}{p-1}\left(p_{i}-1\right)-p_{i}} \frac{\left|x_{i}\right|^{\left(\frac{b_{i}}{b_{1}}-1\right) p_{i}}}{\left[\ln \frac{1}{\sum_{k=1}^{n}\left|x_{k}\right|^{b_{k}}}\right]^{p_{i}}} d x \\
& \leqslant \gamma F_{2}^{p_{i}-1}(r, \lambda), \quad i=1,2, \ldots, n .
\end{aligned}
$$


Оценка интеграла правой части (4.15) получена аналогично (4.9). Из неравенства Гёльдера, леммы 4.1 и (4.15) получаем

$$
\begin{aligned}
I_{3} & \leqslant \gamma \sum_{i=1}^{n}\left(\sum_{j=1}^{n} \int_{E(\rho)} \frac{1}{u(x)}\left|\frac{\partial u}{\partial x_{j}}\right|^{p_{j}} \psi_{r}^{k}(x) d x\right)^{\frac{p_{i}-1}{p_{i}}}\left(\int_{E(\rho)} u^{p_{i}-1}(x)\left|\frac{\partial \psi_{r}(x)}{\partial x_{i}}\right|^{p_{i}} d x\right)^{\frac{1}{p_{i}}} \\
& \leqslant \gamma \sum_{i=1}^{n}\left(\left(\ln \ln \frac{1}{r}\right)^{-\bar{p}_{1}} F_{1}(r, \lambda)+\rho^{p+\lambda} \ln ^{p} \frac{1}{\rho}\right)^{\frac{p_{i}-1}{p_{i}}} F_{2}^{\frac{p_{i}-1}{p_{i}}}(r, \lambda) .
\end{aligned}
$$

Используя неравенство Юнга и (4.5), имеем

$$
\begin{aligned}
& (\widetilde{M}(\rho)-\widetilde{M}(2 \rho))^{1-\varepsilon} I_{4}-\frac{1}{4} \sum_{i=1}^{n} \int_{E(\rho)}(u(x)-\widetilde{M}(2 \rho))^{-\varepsilon}\left|\frac{\partial u}{\partial x_{i}}\right|^{p_{i}} \psi_{r}^{k}(x) d x \\
& \leqslant \gamma(\widetilde{M}(\rho)-\widetilde{M}(2 \rho))^{(1-\varepsilon) p} \int_{E(\rho)} u^{\varepsilon(p-1)}(x) \psi_{r}^{k}(x) d x \\
& \leqslant \gamma(\widetilde{M}(\rho)-\widetilde{M}(2 \rho))^{(1-\varepsilon) p} \rho^{n-\varepsilon(n-p)+\lambda}
\end{aligned}
$$

и

$$
I_{6} \leqslant \gamma \rho^{p+\lambda}
$$

Собирая неравенства (4.14)-(4.18), получим (4.13). Таким образом, лемма 4.2 доказана.

Определим функцию $u^{(\rho)}(x)$ и множество $E(\rho, 2 \rho)$ :

$$
\begin{gathered}
u^{(\rho)}(x)=\min \{(u(x)-\widetilde{M}(2 \rho)), \widetilde{M}(\rho)-\widetilde{M}(2 \rho)\}, \\
E(\rho, 2 \rho)=\{x \in \Omega: \widetilde{M}(2 \rho)<u(x)<\widetilde{M}(\rho)\} .
\end{gathered}
$$

ЛЕмма 4.3. Пусть выполнены все условия леммы 4.1. Тогда справедлива оценка

$$
\sum_{i=1}^{n} \int_{E(\rho, 2 \rho)}\left|\frac{\partial u}{\partial x_{i}}\right|^{p_{i}} d x \leqslant K_{5} \widetilde{M}(\rho) \rho^{\delta+\lambda}\left(1+(\widetilde{M}(\rho)-\widetilde{M}(2 \rho))^{(1-\varepsilon)(p-1)}\right)
$$

с постоянными $\delta>0, K_{5}$, зависящими лищь от $\nu_{1}, \nu_{2}, n, p_{1}, \ldots, p_{n}$.

ДокАЗАТЕЛЬСтво. Подставим в интегральное тождество (2.5) функции

$$
\psi(x)=u^{(\rho)}(x) \psi_{r}^{k-1}(x), \quad \zeta(x)=\psi_{r}(x), \quad k>p_{n} .
$$

Используя неравенства (1.2), получим

$$
\sum_{i=1}^{n} \int_{E(\rho, 2 \rho)}\left|\frac{\partial u}{\partial x_{i}}\right|^{p_{i}} \psi_{r}^{k}(x) d x+\int_{E(2 \rho)} u^{(\rho)}(x) u^{q}(x) \psi_{r}^{k}(x) d x \leqslant \gamma\left(I_{7}+I_{8}+I_{9}+I_{10}\right),
$$


где

$$
\begin{gathered}
I_{7}=\sum_{i=1}^{n} \int_{E(2 \rho)}\left(\sum_{j=1}^{n}\left|\frac{\partial u}{\partial x_{j}}\right|^{p_{j}}\right)^{1-\frac{1}{p_{i}}} u^{(\rho)}(x)\left|\frac{\partial \psi_{r}(x)}{\partial x_{i}}\right| \psi_{r}^{k-1}(x) d x, \\
I_{8}=\int_{E(2 \rho)}\left(\sum_{j=1}^{n}\left|\frac{\partial u}{\partial x_{j}}\right|^{p_{j}}\right)^{1-\frac{1}{p}} u^{(\rho)}(x) \psi_{r}^{k}(x) d x \\
I_{9}=\widetilde{M}(\rho) \sum_{i=1}^{n} \int_{E(2 \rho)} u^{p_{i}-1}(x)\left|\frac{\partial \psi_{r}(x)}{\partial x_{i}}\right|^{p_{i}} d x \\
I_{10}=\widetilde{M}(\rho) \int_{E(2 \rho)} u^{p-1}(x) \psi_{r}^{k}(x) d x .
\end{gathered}
$$

При $\lambda \geqslant 0$ из неравенства Гёльдера и (4.15) получаем

$$
\begin{aligned}
& I_{7} \leqslant \gamma \sum_{i=1}^{n}\left(\sum_{j=1}^{n} \int_{E(2 \rho)} \frac{1}{u(x)}\left|\frac{\partial u}{\partial x_{j}}\right|^{p_{j}} \psi_{r}^{k}(x) d x\right)^{\frac{p_{i}-1}{p_{i}}} \\
& \times\left(\int_{E(2 \rho)} u^{p_{i}-1}(x)\left|\frac{\partial \psi_{r}(x)}{\partial x_{i}}\right|^{p_{i}} d x\right)^{\frac{1}{p_{i}}} \\
& \leqslant \gamma \sum_{i=1}^{n}\left(\sum_{j=1}^{n} \int_{E(2 \rho)} \frac{1}{u(x)}\left|\frac{\partial u}{\partial x_{j}}\right|^{p_{j}} \psi_{r}^{k}(x) d x\right)^{\frac{p_{i}-1}{p_{i}}} F_{2}^{\frac{p_{i}-1}{p_{i}}}(r, \lambda), \\
& I_{8} \leqslant \frac{1}{4} \sum_{i=1}^{n} \int_{E(\rho, 2 \rho)}\left|\frac{\partial u}{\partial x_{i}}\right|^{p_{i}} \psi_{r}^{k}(x) d x+\gamma \widetilde{M}(\rho) \int_{E(\rho, 2 \rho)} u^{p-1}(x) \psi_{r}^{k}(x) d x \\
& \quad+\gamma \widetilde{M}(\rho)\left(\sum_{i=1}^{n} \int_{E(\rho)}(u(x)-\widetilde{M}(2 \rho))^{-\varepsilon}\left|\frac{\partial u}{\partial x_{i}}\right|^{\frac{p-1}{p}} \psi_{r}^{k}(x) d x\right)^{\frac{p-1}{p}} \\
& \quad \times\left(\int_{E(\rho)} u^{\varepsilon(p-1)}(x) \psi_{r}^{k}(x) d x\right)^{\frac{1}{p}} .
\end{aligned}
$$

Собирая неравенства (4.6), (4.11), (4.13), (4.15), (4.18), (4.21), (4.22), из (4.20) получим

$$
\begin{aligned}
& \sum_{i=1}^{n} \int_{E(\rho, 2 \rho)}\left|\frac{\partial u}{\partial x_{i}}\right|^{p_{i}} \psi_{r}^{k}(x) d x \\
& \leqslant \gamma \widetilde{M}(\rho) \rho^{p+\lambda}+\gamma \widetilde{M}(\rho) F_{2}^{p_{1}-1}(r, \lambda)+\gamma \widetilde{M}(\rho) H^{\frac{p-1}{p}}(r, \rho) \rho^{\frac{n-\varepsilon(n-p)+\lambda}{p}} \\
& \quad+\gamma \widetilde{M}(\rho) \sum_{i=1}^{n}\left\{\left(\ln \ln \frac{1}{r}\right)^{-\bar{p}_{1}} F_{1}(r, \lambda)+\rho^{p+\lambda} \ln ^{p} \frac{1}{\rho}\right\}^{\frac{p_{i}-1}{p_{i}}} F_{2}^{\frac{p_{i}-1}{p_{i}}}(r, \lambda)
\end{aligned}
$$

Теперь перейдем к пределу при $r \rightarrow 0$ в неравенстве (4.23).

Равенство

$$
\lim _{r \rightarrow 0} F_{2}(r, \lambda)=0
$$

непосредственно следует из определения функции $F_{2}(r, \lambda)$. 
Также из определения $F_{1}(r, \lambda)$ следует

$$
\begin{array}{lll}
\lim _{r \rightarrow 0} F_{1}(r, \lambda)=0 & \text { при } & \lambda>0, \\
\lim _{r \rightarrow 0}\left(\ln \ln \frac{1}{r}\right)^{-\bar{p}_{1}} F_{1}(r, 0)=0 & \text { при } & q=\frac{n(p-1)}{n-p}, \frac{p_{1}-1}{q-p_{1}+1}=1, \\
\lim _{r \rightarrow 0} F_{1}(r, 0)=0 & \text { при } & q=\frac{n(p-1)}{n-p},
\end{array}
$$

Далее, при $\lambda=0, q=n(p-1) /(n-p),\left(p_{1}-1\right) /\left(q-p_{1}+1\right)<1$ в силу определения $R(r)$ имеем

$$
F_{1}(r, 0) F_{2}(r, 0) \leqslant \gamma\left[\ln \frac{1}{r}\right]^{1-\frac{p_{1}-1}{q-p_{1}+1}-\theta} .
$$

Правая часть (4.28) стремится к нулю при $r \rightarrow 0$, если

$$
\theta>1-\frac{p_{1}-1}{q-p_{1}+1} .
$$

Окончательно, выбирая $\theta$ из условия

$$
\theta= \begin{cases}1-\frac{1}{2} \frac{p_{1}-1}{q-p_{1}+1} & \text { при } \lambda=0, q=\frac{n(p-1)}{n-p}, \frac{p_{1}-1}{q-p_{1}+\lambda}<1, \\ \frac{1}{2} & \text { при } \lambda \geqslant 0, q \geqslant \frac{n(p-1)}{n-p}, \frac{p_{1}-1}{q-p_{1}+1} \geqslant 1,\end{cases}
$$

переходим к пределу при $r \rightarrow 0$ в неравенстве (4.23) и получаем оценку (4.19). Таким образом, лемма 4.3 доказана.

\section{§ 5. Доказательство теоремы 2.1}

Вначале докажем вспомогательную поточечную оценку. Определим функцию $\xi_{\rho}: \mathbb{R}^{1} \rightarrow \mathbb{R}^{1}$ класса $C^{\infty}$ такую, что

$$
\begin{gathered}
\xi_{\rho}(x)=\xi\left(\frac{\left(\sum_{i=1}^{n}\left|x_{i}\right|^{\frac{b_{i}}{b_{1}}}\right)^{b_{1}}}{\rho}\right), \\
\xi(s)= \begin{cases}1 & \text { при } s \geqslant 2, \\
0 & \text { при } s \leqslant 1,\end{cases} \\
0 \leqslant \xi(s) \leqslant 1, \quad\left|\frac{d \xi(s)}{d s}\right| \leqslant \gamma .
\end{gathered}
$$

ЛЕмма 5.1. Предположим, что выполнены все условия теоремы 2.1. Тогда при $0<\rho<R_{0}$ справедлива ощенка

$$
\begin{aligned}
& (\widetilde{M}(\rho)-\widetilde{M}(2 \rho))^{p+n+p_{n} \frac{n}{p}} \\
& \quad \leqslant K_{6} \widetilde{M}^{p_{n} \frac{n}{p}}(\rho)\left[\widetilde{M}^{p}(\rho)+\sum_{i=1}^{n} \widetilde{M}^{p_{i}}(\rho) \rho^{-p-\frac{n-p-\lambda}{p-1}\left(p-p_{i}\right)}\right]^{\frac{n}{p}} \int_{\Omega} u_{2 \rho}^{p}(x) \xi_{\rho}^{p}(x) d x
\end{aligned}
$$

с постоянной $K_{6}$, зависящей лищь от $\nu_{1}, \nu_{2}, n, p_{1}, \ldots, p_{n}$. 
ДокАЗАТЕЛьСтво. Подставим в интегральное тождество (2.5) функции

$$
\psi(x)=u_{2 \rho}^{l+1}(x) \xi_{\rho}^{k-1}(x), \quad \zeta(x)=\xi_{\rho}(x) .
$$

Используя неравенства (1.2), получим

$$
\begin{aligned}
& \sum_{i=1}^{n} \int_{\Omega} u_{2 \rho}^{l}(x)\left|\frac{\partial u}{\partial x_{i}}\right|^{p_{i}} \xi_{\rho}^{k}(x) d x \\
& \quad \leqslant \gamma(l+k)^{p_{n}} H(\rho) \widetilde{M}^{p_{n}}(\rho) \int_{\Omega} u_{2 \rho}^{l-p_{n}}(x) \xi_{\rho}^{k-p_{n}}(x) d x
\end{aligned}
$$

где

$$
H(\rho)=\widetilde{M}^{p}(\rho)+\sum_{i=1}^{n} \widetilde{M}^{p_{i}}(\rho) \rho^{-p-\frac{n-p-\lambda}{p-1}\left(p-p_{i}\right)} .
$$

Используя лемму 2.1 при $\alpha_{1}=\cdots=\alpha_{n}=l(n-p) / n-p$, имеем

$$
\begin{aligned}
& \int_{\Omega} u_{2 \rho}^{l}(x) \xi_{\rho}^{l}(x) d x \\
& \quad \leqslant \gamma\left\{\sum_{i=1}^{n} \int_{\Omega}\left[u_{2 \rho}(x) \xi_{\rho}(x)\right]^{l \frac{n-p}{n}-p}\left|\frac{\partial}{\partial x_{i}}\left(u_{2 \rho}(x) \xi_{\rho}(x)\right)\right|^{p_{i}} d x\right\}^{\frac{n}{n-p}} .
\end{aligned}
$$

Из неравенств (5.2), (5.3), используя итерационный метод Мозера, получим неравенство (5.1). Таким образом, лемма 5.1 доказана.

Отметим, что оценка (2.6) влечет неравенство

$$
\begin{gathered}
\widetilde{M}(\rho) \leqslant \gamma \rho^{-\frac{n-p-\lambda}{p-1}}, \\
\lambda=\frac{(q+1)(n-p)-p(n-1)}{q+1-p} \geqslant 0 .
\end{gathered}
$$

Действительно, если

$$
\left(\sum_{i=1}^{n}\left|x_{i}\right|^{\frac{b_{i}}{b_{1}}}\right)^{b_{1}} \geqslant \rho
$$

то возможны два варианта:

$$
\left|x_{1}\right|>\sum_{i=2}^{n}\left|x_{i}\right|^{\frac{b_{i}}{b_{1}}} ; \quad\left|x_{1}\right| \leqslant \sum_{i=2}^{n}\left|x_{i}\right|^{\frac{b_{i}}{b_{1}}} .
$$

В первом случае из (5.6) имеем $\left|x_{1}\right|^{b_{1}}>2^{-b_{1}} \rho$. Используя теорему 2.2, получим

$$
|u(x)| \leqslant \gamma \rho^{-\frac{p+\frac{n-p-\lambda}{p-1}\left(p-p_{1}\right)}{q-p_{1}+1}}=\rho^{-\frac{n-p-\lambda}{p-1}} .
$$

Во втором случае из (5.6) следует

$$
\left(\sum_{i=2}^{n}\left|x_{i}\right|^{\frac{b_{i}}{b_{1}}}\right)^{b_{1}}>\frac{\rho}{2^{b_{1}}} .
$$


Опять возможны два варианта:

$$
\left|x_{2}\right|>\sum_{i=3}^{n}\left|x_{i}\right|^{\frac{b_{i}}{b_{1}}} ; \quad\left|x_{2}\right| \leqslant \sum_{i=3}^{n}\left|x_{i}\right|^{\frac{b_{i}}{b_{1}}} .
$$

В первом случае из (5.8) имеем $\left|x_{2}\right|^{b_{2}}>2^{-2 b_{1}} \rho$; используя теорему 2.1 , получаем

$$
|u(x)| \leqslant \gamma \rho^{-\frac{p+\frac{n-p-\lambda}{p-1}\left(p-p_{2}\right)}{q-p_{2}+1}}=\rho^{-\frac{n-p-\lambda}{p-1}} .
$$

Во втором случае из (5.8) следует

$$
\left(\sum_{i=3}^{n}\left|x_{i}\right|^{\frac{b_{i}}{b_{1}}}\right)^{b_{1}} \geqslant \frac{\rho}{2^{2 b_{1}}} .
$$

Продолжая рассуждать аналогичным образом, на $n$-м шаге получим

$$
|u(x)| \leqslant \gamma \rho^{-\frac{p+\frac{n-p-\lambda}{p-1}\left(p-p_{n}\right)}{q-p_{n}+1}}=\rho^{-\frac{n-p-\lambda}{p-1}} .
$$

Оценим теперь интеграл в правой части (5.1). Из определения $\widetilde{M}(\rho)$ следует, что

$$
|u(x)| \leqslant M(\rho), \quad \text { если } \xi_{\rho}(x) \neq 0,
$$

поэтому, используя лемму 2.1 при $\alpha_{1}=\cdots=\alpha_{n}=0$, имеем

$$
\begin{aligned}
\int_{\Omega}\left[u_{2 \rho}(x) \xi_{\rho}(x)\right]^{p} d x & \leqslant \int_{\Omega}\left[u^{(\rho)}(x)\right]^{p} d x \leqslant \gamma \rho^{p} \sum_{i=1}^{n} \int_{E(\rho, 2 \rho)}\left|\frac{\partial u}{\partial x_{i}}\right|^{p_{i}} d x \\
& \leqslant \gamma \widetilde{M}(\rho) \rho^{p+\delta+\lambda}\left(1+(\widetilde{M}(\rho)-\widetilde{M}(2 \rho))^{-(\varepsilon-1)(p-1)}\right) .
\end{aligned}
$$

Используя неравенства (5.1), (5.11), получаем

$$
(M(\rho)-M(2 \rho))^{(\varepsilon-1)(p-1)+p+n+p_{n} \frac{n}{p}} \leqslant \gamma \rho^{-\frac{n-p-\lambda}{p-1}\left((\varepsilon-1)(p-1)+n+1+p_{n} \frac{n}{p}\right)-n+p+\lambda+\delta} .
$$

Непосредственными вычисленими из (5.13) получаем

$$
M(\rho)-M(2 \rho) \leqslant \gamma^{-\frac{n-p-\lambda-\delta_{0}}{p-1}}, \quad \delta_{0}=\frac{\delta(p-1)}{(\varepsilon-1)(p-1)+p+n+p_{n} \frac{n}{p}} .
$$

Определим последовательности $\left\{\rho_{j}\right\},\left\{M_{j}\right\}$ равенствами $\rho_{j}=2^{-j} R_{0}, M_{j}=$ $\widetilde{M}\left(\rho_{j}\right)$. Из оценки (5.14) следует

$$
M_{j}-M_{j-1} \leqslant \gamma 2^{j \frac{n-p-\lambda-\delta_{0}}{p-1}} R_{0}^{-\frac{n-p-\lambda-\delta_{0}}{p-1}} .
$$

Суммируя неравенство (5.15) по $2 \leqslant j \leqslant J$, получаем оценку

$$
M_{J}-M_{1} \leqslant \gamma 2^{J \frac{n-p-\lambda-\delta_{0}}{p-1}} R_{0}^{-\frac{n-p-\lambda-\delta_{0}}{p-1}}
$$

из которой следует неравенство

$$
\widetilde{M}(\rho) \leqslant \gamma \rho^{-\frac{n-p-\lambda-\delta_{0}}{p-1}} \quad \text { при } \quad 0<\rho<R_{0} .
$$


При $\lambda=0$, т.е. при $q=n(p-1) /(n-p)$, оценка (5.16) гарантирует равенство (1.8) и, кроме того, включение

$$
|u(x)|^{q-p+1} \in L_{\frac{n}{p-\alpha}}(\Omega), \quad \alpha<\frac{\delta_{0}}{n-p} .
$$

При $\lambda>0$, т.е. при $q>n(p-1) /(n-p)$, рассмотрим

$$
\begin{aligned}
D_{1}^{(j)}(\rho) & =\left\{x \in \Omega:\left(\sum_{i=1}^{n}\left|x_{i}\right|^{\frac{b_{i}\left(\lambda_{j}\right)}{b_{1}\left(\lambda_{j}\right)}}\right)^{b_{1}\left(\lambda_{j}\right)} \leqslant \rho\right\}, \\
M_{1}^{(j)} & =\operatorname{ess} \sup \left\{|u(x)|: x \in D_{1}^{(j)}\left(R_{0}\right) \backslash D_{1}^{(j)}(\rho)\right\}, \\
\lambda_{j} & =\frac{\lambda}{\left(1-\frac{\delta_{0}}{n-p}\right)^{j}}, \quad j=0,1,2, \ldots
\end{aligned}
$$

В силу легко проверяемого неравенства

$$
\frac{n-p-\lambda_{j}}{b_{i}\left(\lambda_{j-1}\right)} \geqslant \frac{n-p-\lambda_{j-1}-\delta_{0}}{b_{i}\left(\lambda_{j}\right)}, \quad i=1,2, \ldots, n, \quad j=1,2, \ldots,
$$

рассуждениями, аналогичными (5.6)-(5.11), можно показать, что из (5.16) следует оценка

$$
M_{1}^{(1)}(\rho) \leqslant \gamma \rho^{-\frac{n-p-\lambda_{1}}{p-1}}
$$

Оценка (5.16) гарантирует последовательное улучшение оценки (5.18) с увеличением $\lambda$ на каждом шаге на положительную величину $\frac{\delta_{0}}{n-p} \lambda /\left(1-\frac{\delta_{0}}{n-p}\right)$ до тех пор, пока $n-p-\lambda_{j}$ остается положительным. За конечное число шагов мы получим неравенство

$$
M_{1}^{(J)} \leqslant \gamma \rho^{-\frac{n-p-\lambda_{J}}{p-1}}
$$

где $\lambda_{J}$ удовлетворяет неравенствам

$$
n-p-\frac{p(p-1)}{q-p+1}<n-p-\frac{\delta_{0}}{2}(n-p)<\lambda_{J}<n-p .
$$

Из (5.19), в частности, имеем

$$
|u(x)|^{q-p+1} \in L_{\frac{n}{p-\alpha}}(\Omega), \quad \alpha<p-\left(n-p-\lambda_{J}\right) \frac{q-p+1}{p-1} .
$$

Кроме того, так как

$$
\frac{n-p-\tilde{\lambda}}{b_{i}\left(\lambda_{J}\right)} \geqslant \frac{n-p-\lambda-\delta_{0}}{a_{i}}, \quad i=1, \ldots, n,
$$

при $\tilde{\lambda}=\lambda_{J}+\delta_{0} / 2$ имеем

$$
\operatorname{ess} \sup \left\{|u(x)|: x \in D\left(R_{0}\right) \backslash D(\rho)\right\} \leqslant \gamma \rho^{-\frac{n-p-\tilde{\lambda}}{p-1}},
$$

где $D(\rho), a_{i}, i=1, \ldots, n$, определены в (1.9), (1.10). Поэтому $u(x)$ удовлетворяет условию (1.8).

Теперь выполнены все условия работы [9], особенность в точке $\{0\}$ устранима. Таким образом, доказательство теоремы 2.1 завершено. 


\section{Список литературы}

[1] H. Brezis, L. Veron, "Removable singularities for some nonlinear elliptic equations", Arch. Rational Mech. Anal, 75:1 (1980), 1-6.

[2] L. Véron, Singularities of solutions of second order quasilinear equations, Pitman Res. Notes Math. Ser., 353, Longman, Harlow, 1996.

[3] И. И. Скрыпник, “Локальное поведение решений квазилинейных эллиптических уравнений с абсорбцией”, Тр. Ин-та матем. и мех. НАН Украинъ, 9 (2004), 183-190.

[4] И.И. Скрыпник, "Устранимость изолированных особенностей решений квазилинейных параболических уравнений с абсорбцией", Матем. сб., 196:11 (2005), 141-160; англ. пер.: I. I. Skrypnik, "Removability of isolated singularities of solutions of quasilinear parabolic equations with absorption", Sb. Math., 196:11 (2005), 1693-1713.

[5] И. М. Колодий, "Об ограниченности обобщенных решений эллиптических дифференциальных уравнений", Вестн. Моск. ун-та. Сер. 1. Матем., мех., 25:5 (1970), 44-52; англ. пер.: I. M. Kolodij, "The boundedness of generalized solutions of elliptic differential equations", Moscow Univ. Math. Bull., 25:5 (1970), 31-37.

[6] N. Fusco, C. Sbordone, "Some remarks on the regularity of minima of anisotropic integrals", Comm. Partial Differential Equations, 18:1-2 (1993), 153-167.

[7] M. Giaquinta, "Growth conditions and regularity, a counterexample", Manuscripta Math., 59:2 (1987), 245-248.

[8] P. Marcellini, Un exemple de solution discontinue d'un problem variationelly dans $l$ cas scalaire, Preprint № 11, Instituto Matematico "Ulisse Dini" Universita di Firenze, Firenze, 1987.

[9] Yu. V. Namlyeyeva, A. E. Shishkov, I. I. Skrypnik, "Isolated singularities of solutions of quasilinear anisotropic elliptic equations", Adv. Nonlinear Stud., 6:4 (2006), 617-641.

[10] F. Nicolosi, I. V. Skrypnik, I. I. Skrypnik, "Precise point-wise growth conditions for removable isolated singularities", Comm. Partial Differential Equations, 28:3-4 (2003), 677-696.

[11] И.В. Скрыпник, Методы исследования нелинейных эллиптических граничных задач, Наука, М., 1990; англ. пер.: I. V. Skrypnik, Methods for analysis of nonlinear elliptic boundary value problems, Transl. Math. Monogr., 139, Amer. Math. Soc., Providence, RI, 1994.

\section{И. И. Скрыпник (I. I. Skrypnik)}

Институт прикладной математики и механики

НАН Украины, г. Донецк

E-mail: iskrypnik@iamm.donbass.com
Поступила в редакцию 14.06.2007 и 25.11.2007 